\title{
A Nonlinear Adaptive Observer-Based Differential Evolution Algorithm to Multiparameter Fault Diagnosis
}

\author{
Xiaoliu Yang $\left(\mathbb{D},{ }^{1}\right.$ Zetao Li $\left(\mathbb{D},{ }^{1}\right.$ Qingfang Zhang, ${ }^{1}$ Qinmu Wu, ${ }^{1}$ and Linli Yang ${ }^{2}$ \\ ${ }^{1}$ College of Electrical Engineering, Guizhou University, Guiyang, Guizhou 550025, China \\ ${ }^{2}$ China University of Mining and Technology, Xuzhou, Jiangshu 221000, China \\ Correspondence should be addressed to Zetao Li; gzulzt@163.com
}

Received 15 September 2019; Revised 6 March 2020; Accepted 26 March 2020; Published 7 May 2020

Academic Editor: Sajad Azizi

Copyright (c) 2020 Xiaoliu Yang et al. This is an open access article distributed under the Creative Commons Attribution License, which permits unrestricted use, distribution, and reproduction in any medium, provided the original work is properly cited.

In this paper, a novel adaptive diagnosis scheme is proposed for multiparametric faults of nonlinear systems by using the model and intelligent optimization-based approaches. The key idea of the proposed method is to analyze the correlation of the output signals between the real system and the fault identification system instead of residual. A new adaptive scheme is built based on an adaptive observer and differential evolution algorithm. Meanwhile, the conditions of detectability and identifiability of faults are analyzed. The isolation and estimation of the multiparametric fault are formulated as the solution of an optimization problem that is solved by using a differential evolutionary algorithm (DE). The fitness function of $\mathrm{DE}$ is constructed by the correlation coefficient equations in which the faulty components are contained. The application on a coupled three water tank model attests the feasibility and validity of the suggested approach. Simulation and experimental results show that the developed method is applicable to diagnose either single or multiparameter faults on-line.

\section{Introduction}

Nonlinearity is considered to be ubiquitous in industrial systems. Due to complicated structures of the systems and harsh working conditions, multifault may coexist in practical applications [1]. In order to improve the safety and reliability of practical systems, prompt detection, robust isolation, and accurate estimation of multifault have become active research areas [2]. Generally, the difficulties of multifault diagnosis mainly originate from two aspects: (1) under severe noise interference, the defect signals generated by different faults will overlap and counteract in the time domain; (2) different faults may excite common or different resonance [1]. Furthermore, the uncertainty and nonlinear characteristics of modern industrial systems have made multifault diagnosis to become a challenging task.

Faults can be classified into catastrophic faults and parametric faults. Catastrophic faults cause the complete failure of the systems. By parametric faults, they mean any deviation in the nominal value implying unwanted changes in the behavior of one or more components of the system.
Parameterization fault is mainly caused by component aging, wear, manufacturing tolerances, and environmental conditions [3]. Degradation of system performance is closely related to the respective parameters. Scientists are prone to believe that efficient diagnosis of small bursts or early failure can prevent rapid degradation of system performance and larger failure [4]. If the parametric faults are not detected and estimated immediately, the system is likely to suffer serious catastrophic faults [5]. The problem of fault diagnosis (FD) thus amounts to the detection and isolation of parameter changes in a model. Multiparametric FD is crucial for the evaluation of fault severity, fault cause analysis, residual life prediction, and fault tolerant control [6]. For these reasons, the focus of this paper considers the multiparametric fault diagnosis based on continuous process monitoring.

Aiming to detect and isolate multiple parametric faults of nonlinear systems, different diagnostic techniques have been investigated. Diagnosis methods may be classified as two types: model-based approach and computationally intelligent-based fault diagnosis methodologies [7]. In modelbased diagnosis, a residual is an indicator of the deviation 
from its expected/normal behavior of a system. A conventional model-based approach is typically relied on a set of residual generators and residual evaluation logic [7]. In an ideal case, a residual generator should be sensitive to a fault. Therefore, the number of possible residual generator candidates grows exponentially with the degree of redundancy of the model [8]. By definition of a residual, a fault-free system means that the ideal output of a residual is zero [9]. However, under the influence of model uncertainty and measurement noise, the residual output of the fault-free system may not be zero, which causes the fault false alarm. To address this problem, much literature has devoted to the thresholding of the residual [10] and the optimization of threshold research [11-13]. In a fault diagnosis system, the objective is not only to develop an algorithm that detects faults as quickly as possible, but also to minimize the false alarm. Therefore, the threshold should be sufficiently small so that low severity faults can be detected in their early stages; on the contrary, thresholds have to be set to sufficiently large values to avoid excessive false alarms due to the presence of noise and uncertainties [7]. In terms of the multiparametric fault estimation for nonlinear systems, optimal selection of multiple threshold bands and designing of multiple observers are still very difficult. For a conventional adaptive observer, the parameter estimation algorithm is formed according to the gradient change of the residual. Due to the coupling of effects of the multiple parameter faults to residual, multiparameter estimation is easy to fall into local optimization.

As the close relative approaches based on intelligent computing have developed rapidly in recent years, evolutionary algorithm, support vector machine algorithm, neural network/deep learning algorithm, and others have been introduced into fault diagnosis methods $[4,7]$. In order to improve the ability of fault parameter estimation in the case of multiparameter faults and nonlinear system, intelligent computing algorithms could be used in the residual analysis process.

In the above types of approach, each has its own advantages and drawbacks. No single method is able to satisfy various diagnostic requirements, especially in the case of nonlinearity, uncertainty, and multiple faults. In recent years, there has been a growing interest in integrating different methods to each other. The development of hybrid approaches could improve the fault diagnosis performances and overcome the limitations of individual methods used separately [14]. Indeed, model-based methods are better for discovery causal relationships, while the methods which are computationally intelligent based are better at finding correlations between faults and symptoms. Therefore, the combination of two methods may potentially derive benefits from each approach and overcome their eventual limitations.

In this paper, a hybrid fault diagnosis scheme is employed. Considering the sensitivity of a residual to a fault, the residual still acts as the basis of fault detection to achieve prompt detection of fault in this paper. To improve the performance of fault isolation, a new adaptive observer based on correlation analysis of the output is built. The system parameters being identified are formulated as the solution of an optimization problem that is solved by using a differential evolutionary (DE) algorithm. The latest identification values of parameters will be sent back to the observer to overcome the uncertainty of the model. It is necessary to mention that different from the usual intelligent computingbased fault diagnosis methods, in this paper, the differential evolution algorithm is not used for the construction of the main framework for fault diagnosis, but for the correlation analysis between the actual system output and the model system output. The fault diagnostic system structure is in principle model based.

The main contributions of this paper are as follows. First, using output correlation coefficient analysis by a differential evolution algorithm, a novel multiparameter fault diagnosis framework is built which is a new construction on an adaptive observer. Secondly, a new scheme is proposed which fuses the advantage of the model-based method in structured analysis and the ability in parameter identification of DE, and the efficiency of fault isolation and identification are improved significantly.

This paper is organized as follows. The considered fault diagnosis problem is formulated in Section 2. The proposed adaptive fault diagnosis scheme-based DE is presented in Section 3. The correlation analysis method and the DE algorithm tool are investigated in Section 4. Validation studies on a benchmark model are addressed in Section 5. Concluding remarks are made in Section 6.

\section{Formulations of System and Fault}

2.1. Nonlinear System Model. Without loss of generality, a parameterized nonlinear system is considered:

$$
M:\left\{\begin{array}{l}
\dot{x}=\varphi(x, t) \theta(t)+B u(t), \\
y=C x,
\end{array}\right.
$$

where $x \in R^{l}, \theta \in R^{p}, u \in R^{m}$, and $y \in R^{n}$ denote the system state, parameter, input vector, and output vector, respectively, $\theta$ is an unknown time-varying parameter, and the term $\varphi(x, t)$ is known as nonlinear functions with appropriate dimensions.

Assumption 1. The uncertain time-varying parameter $\theta(t)$ satisfies

$$
\left|\theta(t)-\theta_{o}\right| \leq \Gamma
$$

where $\theta_{0}$ is known as parameter nominal values and $\Gamma$ is known as a constant vector. Here, the assumed nonlinear system $M$ is linear on parameters. If the process parameter nominal value $\theta_{o}$ is known, the nominal model of the system is

$$
M_{o}:\left\{\begin{array}{l}
\dot{x}=\varphi(x, t) \theta_{o}(t)+B u(t), \\
y_{o}=C x .
\end{array}\right.
$$
follows:

The output error $r(t)$ in the fault detection system is as

$$
r(t)=y(t)-y_{o}(t)
$$


Assumption 2. The fault identification system $M^{\prime}$ has the same model structure with $M$ :

$$
M:=\left\{\begin{array}{l}
\dot{x}^{\prime}=\varphi\left(x^{\prime}, t\right) \theta^{\prime}(t)+B u(t), \\
y^{\prime}=C x^{\prime}
\end{array}\right.
$$

where $y^{\prime}$ is the output of the fault identification system.

2.2. Parametric Fault in Dynamic System. In a system model, a parametric fault is usually related to parameter changes. Therefore, it is natural to monitor the parameter changes to achieve FD. The nominal parameter value $\theta_{0}$ characterizing the fault-free mode of the system is known, and a parametric fault is characterized by a deviation from $\theta_{0}$.

To determine whether the actual system is faulty, the difference between the parameter value $\theta$ of the actual system model $M$ and the parameter value $\theta_{0}$ of the nominal model $M_{o}$ can also be used. When the difference between $\theta$ and $\theta_{0}$ exceeds a certain threshold, it means the system is faulty. Generally, system parameters cannot be measured directly. If $y(t)$ and $y_{o}(t)$ are used to represent the behavior of the actual system and the nominal system, respectively, then the residual $r(t)$ represents the difference between the behavior of the actual system and the one of the nominal system. If the difference $r(t)$ caused by parameter changes exceeds the acceptable level in the view of engineering, it means that there is a fault in the system. Therefore, we can judge whether the system is faulty according to whether the residual $r(t)$ exceeds a certain threshold. The size of the threshold depends on the specific engineering requirements.

\section{Fault Diagnosis Scheme}

3.1. Diagnosability of Multiple Faults of Linear Parametric Variation Model (LPV). Fault diagnosability includes fault detectability, isolability, and identifiability. For multiparameter fault diagnosis, the following definition of fault diagnosability is used to formulate the fault indicator and fitness function selection problem.

Definition 1 (fault detectability). Let $R$ denote a set of residual. A fault mode $F$ is detectable in $R$ if there exists a residual $r_{k} \in R$ that is sensitive to at least one fault $f_{i} \in F$.

Definition 2 (fault isolability). A fault mode $F_{i}$ is isolable from another fault mode $F_{j}$ if there exists a residual $r_{k} \in R$ that is sensitive to at least one fault $f_{i} \in F_{i}$ but not any fault $f_{j} \in F_{j}(i \neq j)$.

Definition 3 (fault identifiability). A fault $f_{i}$ identifiability refers to whether a fault can be uniquely estimated when the input and output data of the model are known.

In this paper, a residual acts as fault test quantity and the output vectors correlation coefficient is used for fault isolation instead of the residual. The correlation coefficient $\rho_{y y^{\prime}}$ of the two output variables can reflect the directivity and waveform change of the two variables and the similarity degree of every unit change. Our approach to fault diagnosis is under the following assumptions: (1) the considered system is component based: a fault $f_{i}$ corresponds to a component. (2) Faults do not change the model structure. (3) The system is observable with its normal working excitation. (4) The parameters are changed at most once in a time window $T$.

Theorem 1. The parameters $\theta$ of a linear parametric variation model can be estimated consistently through the output correlation function if the following necessary conditions are satisfied:

The parameter $\theta_{i}(i=1,2, \ldots, p)$ is distinguishable from each other;

The input signal $u(t)$ is exactly measurable or known; When $t \in\left[t_{0}, t_{0}+T\right]$, the output correlation coefficient $\sum_{i=1}^{n} \rho_{y y^{\prime}}=n$ ( $n$ is the dimension of the output vector $y$ or $y^{\prime}$ ) between the fault detection system and the fault identification system;

The systems' initial state satisfies

$$
x\left(t_{0}\right)=x^{\prime}\left(t_{0}\right)
$$

The systems' state satisfies

$$
x(t)=x^{\prime}(t)
$$

Proof. If $\sum_{i=1}^{n} \rho_{y y^{\prime}}=n$, then the output $y^{\prime}$ is linearly dependent on $y$. Obviously, $y^{\prime}$ can be expressed as

$$
y^{\prime}=K y+D
$$

where $K$ and $D$ are the constant matrix. When $t=t_{0}$, from (3), (5), and (6), there exist

$$
\varphi\left(x, t_{0}\right) \theta\left(t_{0}\right)=\varphi\left(x^{\prime}, t_{0}\right) \theta^{\prime}\left(t_{0}\right) .
$$

Then,

$$
\begin{aligned}
\theta\left(t_{0}\right) & =\theta^{\prime}\left(t_{0}\right), \\
K & =I, \\
D & =0,
\end{aligned}
$$

when $t \neq t_{0}$, and if $x(t)=x^{\prime}(t)$, then $y^{\prime}(t)=y(t)$ such that

$$
\varphi\left(x^{\prime}, t\right) \theta^{\prime}(t)=\varphi(x, t) \theta(t) .
$$

Since $x(t)=x^{\prime}(t)$, then we have

$$
\theta^{\prime}(t)=\theta(t)
$$

The proof is complete.

The above proof means that the precise estimation of the parameters can be obtained if the output curves of two systems overlap, respectively, within a time window. Accordingly, the coordinates of the parameters in the parameter space and the fault mode (single fault or multiple faults) can be determined. Therefore, the fault identification problem can be solved as an optimization problem. The optimization problem is as follows: 


$$
\begin{array}{ll}
\min & \left(\sum_{i=1}^{n} \rho_{y y^{\prime}}-n\right), \\
\text { s.t. } & \theta_{\min } \leq \theta \leq \theta_{\max },
\end{array}
$$

where $n$ denotes the output dimensions which is known for a certain system model and the correlation coefficient $\rho_{y, y^{\prime}}$ is the optimised variable. This diagnosis method can be seen as a parameter identification problem in engineering since the input and the output are known, and it is used to estimate the parameters of the model.

3.2. Fault Detection Based on Luenberger-Like Observer. Considering a system of the form (1), a Luenberger-like observer acts as an estimator of the state $x(t)$ from the measurable $u(t)$ and $y(t)$. The state observer can ensure that the estimated state keeps track of the system state in time. When parameter $\theta$ subjects to changes, the residual between the estimated output and the system output is clearly not equal to 0 . Thus, the fault is detected. For fault detection, a parameter vector of the nominal system is used:

$$
\left\{\begin{array}{l}
\dot{\hat{x}}=\varphi(\widehat{x}, t) \theta_{0}+B u(t)+K_{\mathrm{obv}}(y-\hat{y}), \\
\hat{y}=C \hat{x}
\end{array}\right.
$$

where $\hat{x}$ is the estimate of $x$ and $K_{\text {obv }}$ represents the gain of the fault detection observer.

Under certain assumptions about the system structure and input excitation, and if the gain vector $K_{\mathrm{obv}}$ is chosen appropriately, for example, according to Lyapunov theorem, the estimated state vector can converge to its actual value. Thus, the necessary condition (5) of Theorem 1 can be ensured. There are many ways to obtain the observer gain matrices, but we will not deal with this problem in this paper. The interested reader is referred to [13].

Note: when system (1) is deterministic, the estimation of $x(t)$ amounts in principle to the estimation of the initial state $x\left(t_{0}\right)$. However, due to modeling errors, it makes sense to estimate the trajectory $x(t)$ instead of the initial state $x\left(t_{0}\right)$.

Residual of output variables: $r(t)=\hat{y}-y$ is used for fault detection. If $r(t)=0$, it means that the actual system is fault free. If the absolute value of $r(t)$ is greater than a preset threshold, the system is determined faulty. Of course, in the case of considering system uncertainty, environmental noise, and other factors, the residual threshold will be more complex. Because the fault detection is not the main topic of this paper, interested readers please refer to the literature [13].

3.3. Fault Isolation and Identification Scheme. In the parameter estimation-based fault diagnosis method, if the parameter vector value of the actual dynamic system is identified, the parameter component in the actual system parameter vector which is different relative to the one in the normal system parameter vector, i.e., the faulty parameter, is found, so the fault is isolated. At the same time, the magnitude of the deviation of the faulty parameter from the normal value is estimated, so the fault is identified.
As an initial motivation, a conventional Luenberger-like adaptive observer is designed to estimate the states and parameters. It is presented as follows:

$$
\left\{\begin{array}{l}
\dot{\hat{x}}=\varphi(\widehat{x}, t) \hat{\theta}+B u(t)+K_{\mathrm{obv}}(y-\widehat{y}), \\
\hat{y}=C \hat{x}, \\
\dot{\hat{\theta}}=K_{\theta}(y-\hat{y}),
\end{array}\right.
$$

where $\hat{\theta}$ represents the parameter estimates which are composed of parameters in which may occur faults according to prior knowledge and $K_{\theta}$ denotes the gain of the parameter estimation observer. The state estimates the equation in the above observer and still uses an output variable residual $r(t)$ for state correction. The objective of selecting the gain $K_{\mathrm{obv}}$ in the state estimate equation is to ensure the sensitivity of the output residual of the parameter deviation $\theta-\widehat{\theta}$. That is to say, if parameter deviation $\theta-\widehat{\theta} \neq 0$, then the output residual $r(t) \neq 0$. On the contrary, the selecting of the gain $K_{\text {obv }}$ should ensure that output residual $r(t)$ and state error $x(t)-\hat{x}(t)$ have certain robustness to noise, uncertainty, initial condition difference, etc. That is to say, when the parameter deviation $\theta-\widehat{\theta}=0$, the gain $K_{\mathrm{obv}}$ can eliminate the effects of noise, uncertainty, and initial condition differences and make the output residual and the state error converge to zero.

In the above Luenberger-like adaptive observer, the parameter identification of the Luenberger-like adaptive observer chooses the search path according to the variation of the output variable output continuously. However, for a nonlinear dynamic system, especially in the case of multiparameters, in the continuous parameter identification search path, the case is often complex and difficult. There may be local optimal points or flat road sections which cause the failure of parameter identification. To overcome the disadvantages of this kind of continuous path search method, the differential evolution (DE) algorithm-based relative analysis can be used to fulfill parameter estimation in the adaptive observer. Consequently, the following DEbased adaptive observer is obtained:

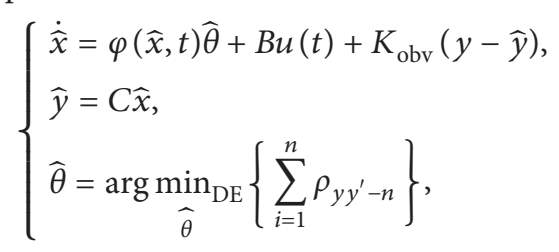

where $\min _{\widehat{\theta}}\{*\}$ presents the optimization calculation using the differential evolution algorithm, $\rho_{y y^{\prime}}$ is a correlation coefficient which measures the degree of similarity between different signals, and the absolute value of $\rho_{y y^{\prime}}$ is larger when the degree of correlation between two time series is higher.

The objective function of DE optimization calculation is chosen as $\min \left(\sum_{i=1}^{n} \rho_{y y^{\prime}}-n\right)$. The choice of this objective function is based on the consideration that the correlation calculation only analyzes the morphological similarity between the variables and does not care about the difference in the amplitude of the variables. 
To sum up, the fault diagnosis scheme based on an adaptive observer using DE is shown in Figure 1. Figure 1 shows the scheme of the fault diagnosis as a solution of an optimization problem. This article will use the above scheme of fault diagnosis based on parameter estimation in a nonlinear system. Such approach is presented in the next section.

\section{Mathematical and Algorithm Tools}

4.1. Correlation Analysis. As a statistical method, correlation analysis has been applied in evaluating the strength of the relationship between two quantitative variables with available statistical data. This technique is strictly connected to the linear regression analysis that is a statistical approach for modeling the association between a dependent variable and one or more explanatory or independent variables. In this paper, the Pearson correlation coefficient is chosen to extract the feature and apply it to multifault identification. This method has many advantages, such as high accuracy, high efficiency, and strong practicability.

The Pearson correlation coefficient presents a measure of how two random variables relate linearly in a sample. The $Y$ and $Y$, vectors in the following formula define the residual correlation coefficient, which is generally denoted by the symbol $\rho_{Y Y^{\prime}}$ :

$$
\rho_{Y Y^{\prime}}=\frac{\operatorname{cov}\left(Y, Y^{\prime}\right)}{\sigma_{Y} \sigma_{Y^{\prime}}}=\frac{E\left(Y Y^{\prime}\right)-E(Y) E\left(Y^{\prime}\right)}{\sqrt{E\left(Y^{2}\right)-E^{2}(Y)} \times \sqrt{E\left(Y^{\prime 2}\right)-E^{2}\left(Y^{\prime}\right)}},
$$

where cov is the covariance, $\sigma$ is the standard deviation, and $E$ is the expectation. The Pearson correlation coefficient lies in the range $-1 \leqslant \rho_{Y Y^{\prime}} \leqslant 1$. In order to obtain the correlation coefficient, obtaining $\sigma_{Y^{\prime}}, \sigma_{Y}$, and $\operatorname{cov}\left(Y, Y^{\prime}\right)$ is necessary. In practice, these parameters for the variables are either unknown or difficult to obtain. Thus, the alternative is using $\rho_{Y Y^{\prime}}$ which one can be obtained from a sample. The following formula expresses the sample correlation coefficient $\rho_{Y Y^{\prime}}$ :

$$
\rho_{Y Y^{\prime}}=\frac{\sum_{i=1}^{T / T_{s}}\left(y_{i}^{\prime}-\overline{y^{\prime}}\right)\left(y_{i}-\bar{y}\right)}{\sqrt{\sum_{i=1}^{T / T_{s}}\left(y_{i}^{\prime}-\overline{y^{\prime}}\right)^{2}} \sqrt{\sum_{i=1}^{T / T_{s}}\left(y_{i}-\bar{y}\right)^{2}}},
$$

where

$$
\overline{y^{\prime}}=\frac{1}{T / T_{s}} \sum_{i=1}^{T / T_{s}}\left(y_{i}^{\prime}\right), \bar{y}=\frac{1}{T / T_{s}} \sum_{i=1}^{T / T_{s}}\left(y_{i}\right)
$$

in which $T$ is the length of the data window used for fault identification; $T_{s}$ is the sampling step size; $\left(y_{i}^{\prime}, y_{i}\right)$ is the $i_{\text {th }}$ pair observation value; and $\overline{y^{\prime}}$ and $\bar{y}$ are sample means for $y^{\prime}$ and $y$, respectively. Pearson correlation is a straightforward approach to evaluate the relationship between two variables. The larger the $|r|$ is, the more significant the relationship between $Y$ and $Y^{\prime}$ is.

In the subject of signal and system, correlation analysis is an important method to describe signal characteristics in the time domain. Since the correlation concepts were introduced to study the statistical characteristics of random signals, they can be also theoretically applied to the study of the similarity between two deterministic signals (one of the reference waveform and one of the signal waveform collected).

4.2. Differential Evolution Algorithm (DE). Differential evolution (DE) is a simple and powerful intelligent optimization algorithm. As an advanced version of the genetic algorithm (GA), DE has been the subject of much attention due to its attractive characteristics of the need for lower parameters, simple structure, ease of use, and robustness $[15,16]$. DE has been extended for handling multiobjective, constrained, large-scale, dynamic, and uncertain optimization problems. Due to the advantages of DE, it was developed for many applications in engineering fields. In DE, a general description includes four operators of population initialization, mutation, crossover, and selection. An overview of the main steps of the $\mathrm{DE}$ algorithm is presented next.

4.2.1. Initialization. Setting up the maximum generation number $G_{\max }$ and generation index $G=1$, the initial population within the range $\left[\theta_{i}^{\min }, \theta_{i}^{\max }\right]$ of parameters is randomly created.

$$
\theta_{i}=\theta_{i}^{\min }+\operatorname{rand}(0,1) \times\left(\theta_{i}^{\max }-\theta_{i}^{\min }\right),
$$

where rand $(0,1)$ denotes a random number in the interval $(0,1), i=1,2, \ldots, N P$. The value of NP is the number of individuals of population that will affect computational time and fault identification performance. Increasing NP means high computational time but low risk to be trapped in local minimum.

4.2.2. Mutation. Mutant individual is generated by using (21). The scale factor of difference perturbation $F$ is chosen in the range of $[0,2]$.

$$
\theta_{G}=\theta_{j}+F \times\left(\theta_{k}-\theta_{l}\right),
$$

where $\theta_{j}, \theta_{k}, \theta_{l}(j \neq k \neq l)$ are randomly selected vectors, $\theta_{G}$ is a mutant, and all of these vectors must be dissimilar so that the population contains at least four individuals.

\subsubsection{Crossover}

$$
\theta_{t}= \begin{cases}\theta_{G}, & \text { if rand }(0,1) \leq \mathrm{CR} \text { or } j=q, \\ \theta_{i}, & \text { otherwise, }\end{cases}
$$

where $j=1,2, \ldots, D$ and $q \in[1,2, \ldots, D]$ is a random index. The value of CR is chosen in the range of $[0,1]$.

4.2.4. Selection Operation. Fitness computation $f(\theta)$ by (13) and comparison by (23) is used to update the population.

$$
\theta_{t+1}= \begin{cases}\theta_{t}, & \text { if } f\left(\theta_{t}\right)<f\left(\theta_{i}\right) \\ \theta_{i}, & \text { otherwise. }\end{cases}
$$




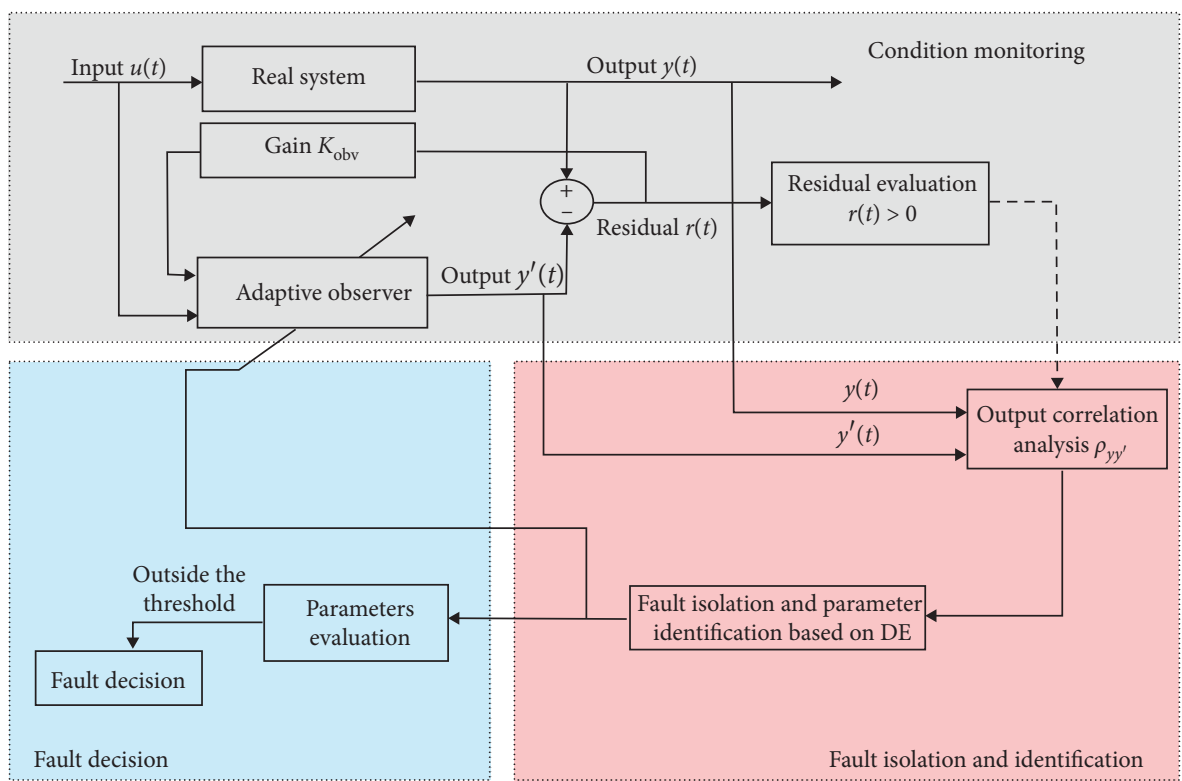

FIgURe 1: A multiparameter fault diagnosis scheme of a nonlinear adaptive observer based on DE.

This step is repeated until the termination criterion is satisfied, and hence a population of solution is obtained.

\section{Case Studies}

The effectiveness of the proposed correlation-based robust FDI is demonstrated through typical nonlinear dynamic process systems with variable parameters.

5.1. Description of Three-Tank Model. The three-tank system is widely used for comparison and demonstration purpose in control engineering as a benchmark process in laboratories for process control [17]. To illustrate the effectiveness of the proposed methodology, in this section, a simulation experiment on the three-tank system (TTS) is introduced. TTS is shown in Figure 2. The precise mathematical model can easily describe the multiply faults such as leakage/plugging/ sensor/actuator faults. Industrial systems consist of the plant (or system dynamics), sensors, and actuators [4]. The sensor and actuator faults are additive fault. The leakage and plugging faults are usually nonadditive. In the references, many efficient fault estimation methods of the sensors and the actor can be found [18-21]. However, from the FTC point of view, much more important is the problem of the plant (component) fault diagnosis. There have been few studies on nonadditive faults, especially for the nonlinear dynamic system. In this paper, two types of tank faults (leakage and plugging faults) are belonging to nonadditive faults.

Applying the incoming and outgoing mass flows with the Torricellies law, the dynamics of TTS is modeled by

$$
\left\{\begin{array}{l}
\frac{\mathrm{d} h_{1}}{\mathrm{~d} t}=\frac{1}{A}\left(Q_{1}-\delta_{1} A_{n} \operatorname{sgn}\left(h_{1}-h_{3}\right) \sqrt{2 g\left|h_{1}-h_{3}\right|}\right), \\
\frac{\mathrm{d} h_{2}}{\mathrm{~d} t}=\frac{1}{A}\left(Q_{2}+\delta_{3} A_{n} \operatorname{sgn}\left(h_{3}-h_{2}\right) \sqrt{2 g\left|h_{3}-h_{2}\right|}-\delta_{2} A_{n} \sqrt{2 g h_{2}}\right), \\
\left.\frac{\mathrm{d} h_{3}}{\mathrm{~d} t}=\frac{1}{A}\left(\delta_{1} A_{n} \operatorname{sgn}\left(h_{1}-h_{3}\right) \sqrt{2 g\left|h_{1}-h_{3}\right|}-\delta_{3} A_{n} \operatorname{sgn}\left(h_{3}-h_{2}\right) \sqrt{2 g\left|h_{3}-h_{2}\right|}\right)\right),
\end{array}\right.
$$

where $A$ is the cross-section area of the tank; $A_{n}$ is the crosssection area of the pipe; $Q_{1}, Q_{2}$ are the incoming mass flow $\left(\mathrm{cm}^{3} / \mathrm{s}\right) ; h_{i}(t), i=1,2,3$, are the water level $(\mathrm{cm})$ of each tank and measured; $\delta_{i}, i=1,2,3$ is the coefficient of flow for pipes.

The state vector $X$, output vector $Y$, and input $U$ will be

$$
\begin{aligned}
X & =\left[h_{1}, h_{2}, h_{3}\right]^{T} \in R^{3}, \\
Y & =\left[h_{1}, h_{2}, h_{3}\right]^{T}, \\
U & =\left[Q_{1}, Q_{2}\right]^{T} \in R^{2}, \\
Q_{1} & =Q_{2} .
\end{aligned}
$$




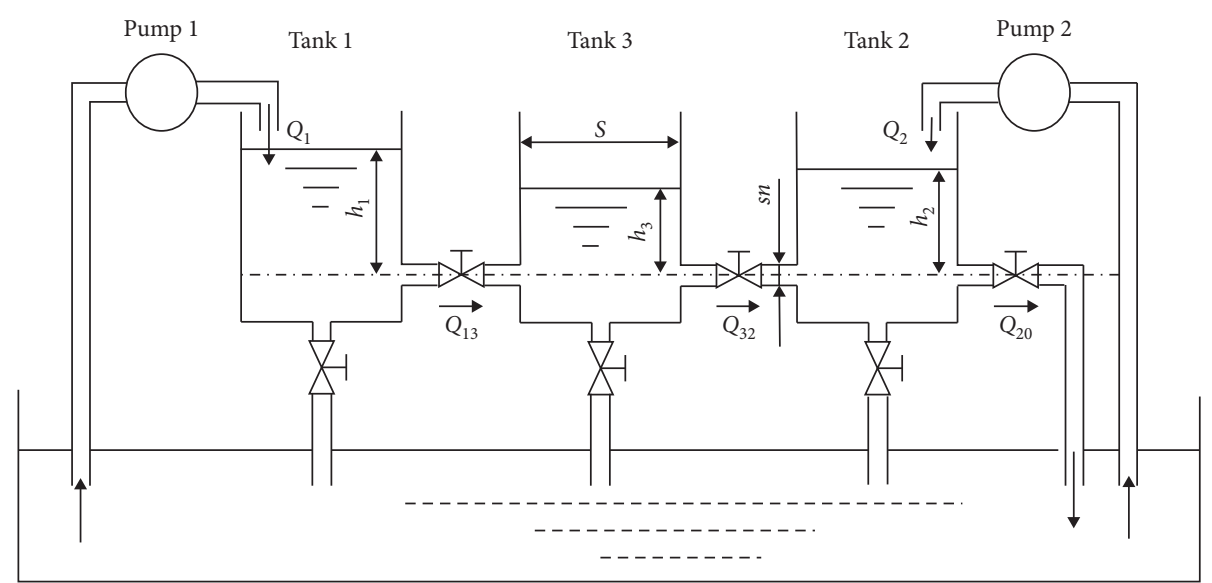

Figure 2: Three-tank system by Ding [22].

$$
\begin{aligned}
& \left\{\begin{array}{l}
\frac{\mathrm{d} x_{1}}{\mathrm{~d} t}=\frac{1}{A}\left(Q_{1}-\delta_{1} A_{n} \operatorname{sgn}\left(x_{1}-x_{3}\right) \sqrt{2 g\left|x_{1}-x_{3}\right|}\right), \\
\frac{\mathrm{d} x_{2}}{\mathrm{~d} t}=\frac{1}{A}\left(Q_{2}+\delta_{3} A_{n} \operatorname{sgn}\left(x_{3}-x_{2}\right) \sqrt{2 g\left|x_{3}-x_{2}\right|}-\delta_{2} A_{n} \sqrt{2 g x_{2}}\right), \\
\left.\frac{\mathrm{d} x_{3}}{\mathrm{~d} t}=\frac{1}{A}\left(\delta_{1} A_{n} \operatorname{sgn}\left(x_{1}-x_{3}\right) \sqrt{2 g\left|x_{1}-x_{3}\right|}-\delta_{3} A_{n} \operatorname{sgn}\left(x_{3}-x_{2}\right) \sqrt{2 g\left|x_{3}-x_{2}\right|}\right)\right),
\end{array}\right. \\
& Y=\left[x_{1}, x_{2}, x_{3}\right]^{T}, x\left(t_{0}\right)=x_{0}, u_{1}=Q_{1}=Q_{2} .
\end{aligned}
$$

5.2. Multifault Diagnosis Results. In this paper, the process is affected by two types of faults: the leak faults and the plugging faults. The leaks in the three tanks can be modeled as additional outgoing mass flows of tanks [22]:

$$
\begin{aligned}
& Q_{f 1}=a_{1} S_{1} \sqrt{2 g x_{1}}, \\
& Q_{f 2}=a_{2} S_{2} \sqrt{2 g x_{2}}, \\
& Q_{f 3}=a_{3} S_{3} \sqrt{2 g x_{3}},
\end{aligned}
$$

where $S_{1}, S_{2}$, and $S_{3}$ are the cross-sectional area of leaks, which are unknown and depend on the size of the leaks, and $a_{1}, a_{2}$, and $a_{3}$ are the coefficients of flow for leaks and unknown. The plugging between two tanks and in the outlet pipe by tank 2 can be modeled by

$$
\left\{\begin{array}{l}
Q_{f 4}=b_{1} \delta_{1} A_{n} \operatorname{sgn}\left(x_{1}-x_{3}\right) \sqrt{2 g\left|x_{1}-x_{3}\right|}, \\
Q_{f 5}=b_{3} \delta_{3} A_{n} \operatorname{sgn}\left(x_{3}-x_{2}\right) \sqrt{2 g\left|x_{3}-x_{2}\right|} \\
Q_{f 6}=b_{2} \delta_{2} A_{n} \sqrt{2 g x_{2}},
\end{array}\right.
$$

where $b_{1}, b_{2}, b_{3} \in[0,1]$ are unknown. The model of plant faults is modeled by

$$
\begin{aligned}
& \left\{\begin{array}{l}
\dot{x}_{1}=\frac{1}{A}\left(u_{1}-\delta_{1} A_{n} \operatorname{sgn}\left(x_{1}-x_{3}\right) \sqrt{2 g\left|x_{1}-x_{3}\right|}+Q_{f 4}-Q_{f 1}\right), \\
\dot{x}_{2}=\frac{1}{A}\left(u_{1}+\delta_{3} A_{n} \operatorname{sgn}\left(x_{3}-x_{2}\right) \sqrt{2 g\left|x_{3}-x_{2}\right|}-\delta_{2} A_{n} \sqrt{2 g x_{2}}-Q_{f 5}+Q_{f 6}-Q_{f 2}\right), \\
\dot{x}_{3}=\frac{1}{A}\left(\delta_{1} A_{n} \operatorname{sgn}\left(x_{1}-x_{3}\right) \sqrt{2 g\left|x_{1}-x_{3}\right|}-\delta_{3} A_{n} \operatorname{sgn}\left(x_{3}-x_{2}\right) \sqrt{2 g\left|x_{3}-x_{2}\right|}-Q_{f 4}+Q_{f 5}-Q_{f 3}\right) .
\end{array}\right. \\
& \begin{array}{l}
\text { In general, the size of the leaks is less than the pipes, then } \\
\begin{array}{l}
a_{i}=\rho_{i} \delta_{i}, \\
S_{i}=k_{i} S_{n}, \\
i=1,2,3,
\end{array}
\end{array}
\end{aligned}
$$
the coefficient of flow for leaks is less than that of the pipes, thus let 
TABle 1: Process parameters of the system.

\begin{tabular}{lccc}
\hline Parameters & Symbol & Value & Unit \\
\hline Cross-section area of tanks & A & 160 & $\mathrm{~cm}^{2}$ \\
Cross-section area of pipes & $A_{n}$ & 0.6 & 100 \\
Max height of tanks & $H_{\max }$ & 90 & $\mathrm{~cm}^{2}$ \\
Max flow rate of pump 1 & $Q_{1 \max }$ & 90 & $\mathrm{~cm}^{3} / \mathrm{s}$ \\
Max. flow rate of pump 2 & $Q_{2 \max }$ & 0.6 & $\mathrm{~cm}^{3} / \mathrm{s}$ \\
Coefficient of flow for pipe 1 & $\delta_{1}$ & 0.6 & 0.6 \\
Coefficient of flow for pipe 2 & $\delta_{2}$ & $\delta_{3}$ & \\
Coefficient of flow for pipe 3 & $\delta_{3}$ & 0.6 & \\
\hline
\end{tabular}

TABle 2: Diagnosis results with different fault dimension

\begin{tabular}{|c|c|c|c|c|c|c|c|c|c|c|c|}
\hline Fault cases & $\theta_{1}$ & $\hat{\theta}_{1}$ & $\theta_{2}$ & $\widehat{\theta}_{2}$ & $\theta_{3}$ & $\widehat{\theta}_{3}$ & $\theta_{4}$ & $\widehat{\theta}_{4}$ & $\theta_{5}$ & $\widehat{\theta}_{5}$ & $\rho_{y y^{\prime}}$ \\
\hline 1-dimention & 0.1 & 0.099672 & 1 & 0.99895 & 0 & 0.002993 & 1 & 0.988149 & 1 & 0.997931 & 3 \\
\hline 2-dimention & 0.1 & 0.090868 & 0.6 & 0.601092 & 0 & 0.007281 & 1 & 0.978094 & 1 & 0.999205 & 3 \\
\hline 3-dimention & 0.1 & 0.098079 & 0.6 & 0.598053 & 0.5 & 0.598053 & 1 & 0.994553 & 1 & 0.996132 & 3 \\
\hline 4-dimention & 0.1 & 0.11311 & 0.6 & 0.598932 & 0.5 & 0.483429 & 0.7 & 0.742294 & 1 & 0.996307 & 3 \\
\hline 5-dimention & 0.1 & 0.096488 & 0.6 & 0.606524 & 0.5 & 0.513945 & 0.7 & 0.689603 & 0.9 & 0.912194 & 3 \\
\hline
\end{tabular}

where $\rho_{i}, k_{i}$ are the scale factors and $\rho_{i}, k_{i} \in[0,1]$.

Note: as shown by equations (27) and (28), fault 2 and fault 6 are linearly correlated with respect to $\sqrt{2 g x_{2}}$, which does not satisfy the first necessary condition of Theorem 1 . Therefore, fault 2 and fault 6 cannot be accurately identified and isolated at the same time in this system. The defining $Q_{f 2}^{*}=Q_{f 6}-Q_{f 2}$ is a trade-off in order to achieve a linear description on parameters. The linear parameter-space representation then becomes

$$
\left\{\begin{array}{l}
\dot{x}_{1}=\frac{1}{A}\left(u_{1}-\theta_{4} \delta_{1} A_{n} \operatorname{sgn}\left(x_{1}-x_{3}\right) \sqrt{2 g\left|x_{1}-x_{3}\right|}\right)-\theta_{1} \delta_{1} A_{n} \sqrt{2 g x_{1}}, \\
\dot{x}_{2}=\frac{1}{A}\left(u_{1}+\theta_{5} \delta_{3} A_{n} \operatorname{sgn}\left(x_{3}-x_{2}\right) \sqrt{2 g\left|x_{3}-x_{2}\right|}+\theta_{2} \delta_{2} A_{n} \sqrt{2 g x_{2}}\right) \\
\dot{x}_{3}=\frac{1}{A}\left(\theta_{4} \delta_{1} A_{n} \operatorname{sgn}\left(x_{1}-x_{3}\right) \sqrt{2 g\left|x_{1}-x_{3}\right|}-\theta_{5} \delta_{3} A_{n} \operatorname{sgn}\left(x_{3}-x_{2}\right) \sqrt{2 g\left|x_{3}-x_{2}\right|}-\theta_{3} \delta_{3} A_{n} \sqrt{2 g x_{3}}\right),
\end{array}\right.
$$

where $\theta_{1}=\rho_{1} k_{1}, \theta_{2}=\rho_{2} k_{2}-1+b_{2}, \theta_{3}=\rho_{3} k_{3}, \theta_{4}=1-b_{1}$, $\theta_{5}=1-b_{3}$. The nominal values of parameters are $\theta_{1}^{0}=0, \theta_{2}^{0}=1, \theta_{3}^{0}=0, \theta_{4}^{0}=1, \theta_{5}^{0}=1$. The range of the parameters are $\theta_{i} \in[0,1](i=1,3,4,5)$ and $\theta_{2} \in[-1,1]$. Equation (31) is multiple linear regression equations and can be transformed into the following linear parameter-varying form:

$$
\dot{x}=F(x) \theta+B u,
$$

where

$$
\begin{aligned}
& F(x)=\left[\begin{array}{ccccc}
-\delta_{1} A_{n} \sqrt{2 g x_{1}} & 0 & 0 & -\delta_{1} A_{n} \operatorname{sgn}\left(x_{1}-x_{3}\right) \sqrt{2 g\left|x_{1}-x_{3}\right|} & 0 \\
0 & \delta_{2} A_{n} \sqrt{2 g x_{2}} & 0 & 0 & \delta_{3} A_{n} \operatorname{sgn}\left(x_{3}-x_{2}\right) \sqrt{2 g\left|x_{3}-x_{2}\right|} \\
0 & 0 & -\delta_{3} A_{n} \sqrt{2 g x_{3}} & \delta_{1} A_{n} \operatorname{sgn}\left(x_{1}-x_{3}\right) \sqrt{2 g\left|x_{1}-x_{3}\right|} & -\delta_{3} A_{n} \operatorname{sgn}\left(x_{3}-x_{2}\right) \sqrt{2 g\left|x_{3}-x_{2}\right|}
\end{array}\right], \\
& B=\left[\frac{1}{A}, \frac{1}{A}, 0\right] .
\end{aligned}
$$




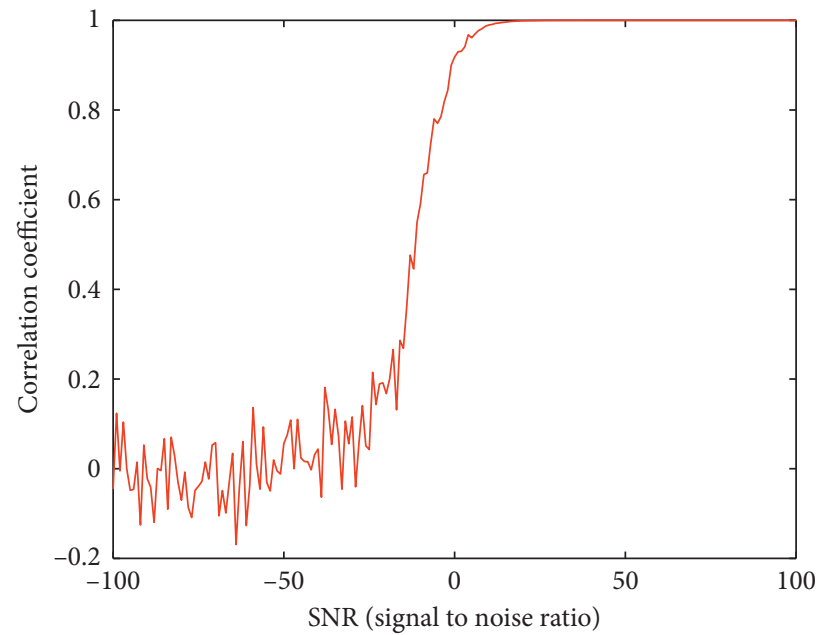

FIgURE 3: The relationship between the correlation coefficient and signal to noise ratio (SNR).

TABLE 3: The parameter estimation results with different SNR.

\begin{tabular}{lcccccc}
\hline Parameter & Fault value & SNR $=50$ & SNR $=40$ & SNR $=30$ & SNR $=20$ & SNR $=10$ \\
\hline$\theta_{1}$ & 0.3 & 0.300550 & 0.298721 & 0.290431 & 0.307865 & 0.275272 \\
$\theta_{2}$ & 0.3 & 0.301163 & 0.259900 & 0.295185 & 0.274000 & 0.303014 \\
$\theta_{3}$ & 0.5 & 0.491633 & 0.494183 & 0.495723 & 0.493756 & 0.428642 \\
$\theta_{4}$ & 0.6 & 0.602957 & 0.603952 & 0.594899 & 0.595272 & 0.616803 \\
$\theta_{5}$ & 1.0 & 0.996162 & 0.991430 & 0.998303 & 0.998101 & 0.995124 \\
\hline
\end{tabular}
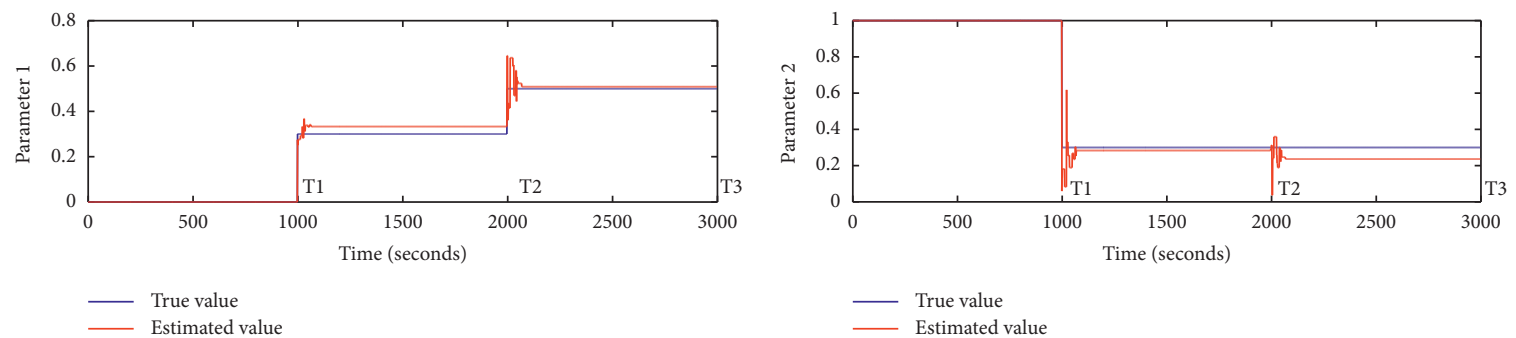

(a)

(b)
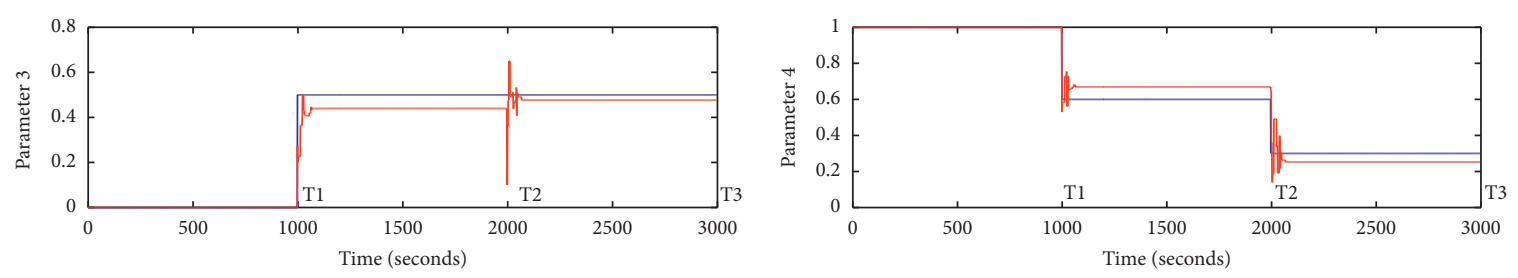

True value
Estimated value

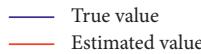

(c)

(d)

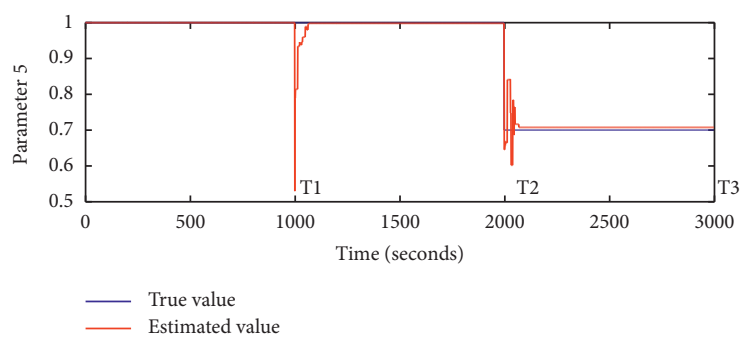

(e)

FIGURE 4: Estimation of the five parameters $(\mathrm{SNR}=10)$. 


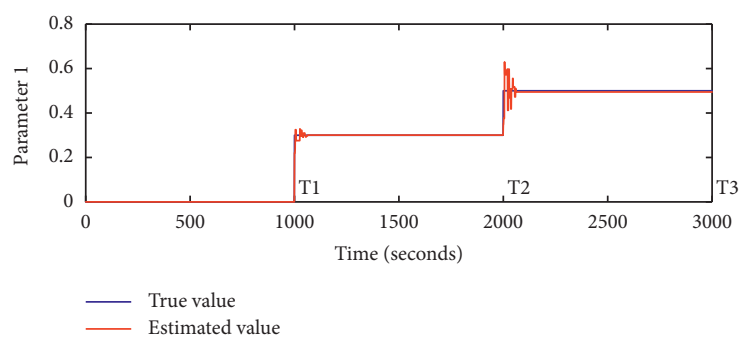

(a)

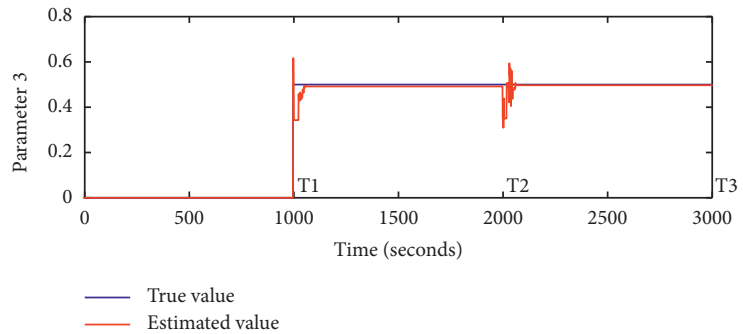

(c)

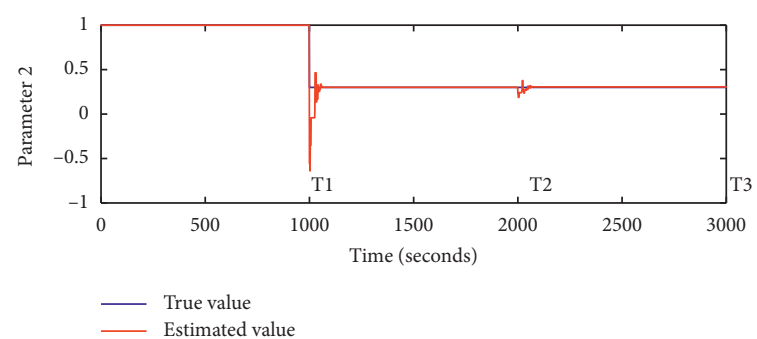

(b)

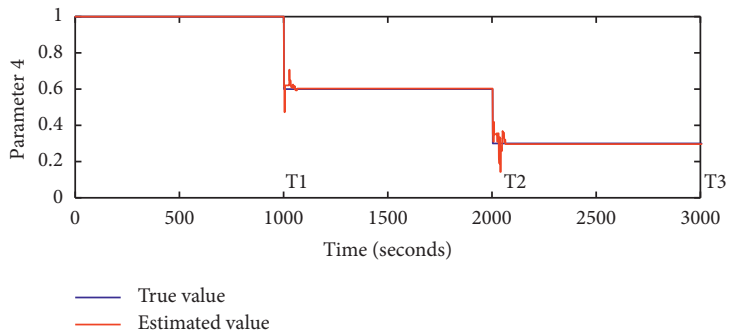

(d)

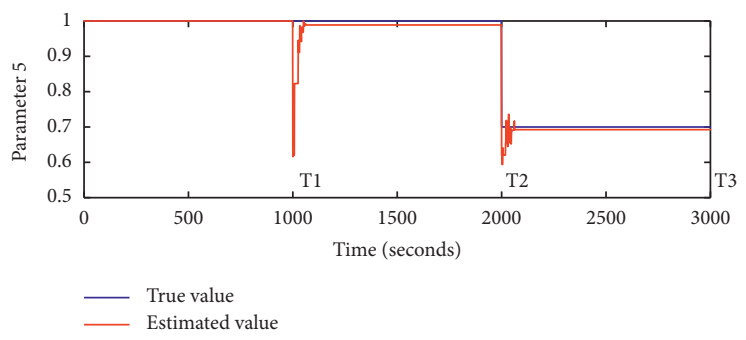

(e)

FIgURE 5: Estimation of the five parameters $(\mathrm{SNR}=50)$.

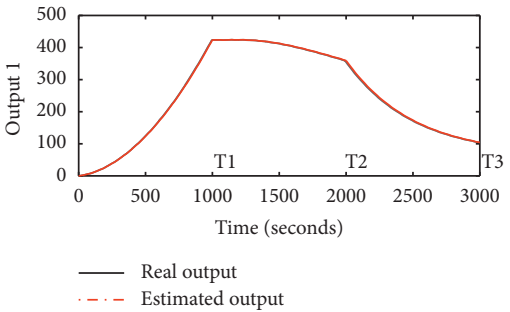

(a)

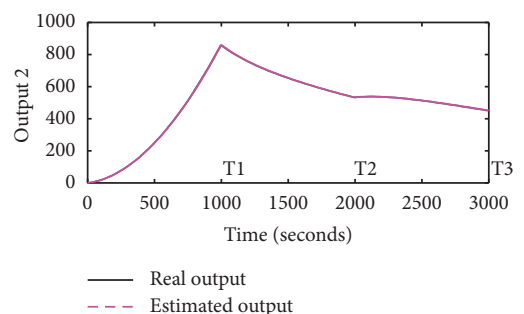

(b)

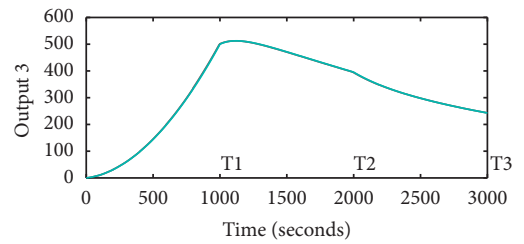

_... Estimated output

(c)

Figure 6: The residual output (time window $=1000, \mathrm{SNR}=50$ ).

The output residuals $r_{1}(t), r_{2}(t)$, and $r_{3}(t)$ of the fault detection system are shown as follows:

$$
\begin{aligned}
& r_{1}(t)=y_{1}-y_{1}^{\prime}, \\
& r_{2}(t)=y_{2}-y_{2}^{\prime}, \\
& r_{3}(t)=y_{1}-y_{3}^{\prime},
\end{aligned}
$$

where $y_{1}, y_{2}$, and $y_{3}$ are the system outputs and $y_{1}^{\prime}, y_{2}^{\prime}$, and $y_{3}^{\prime}$ are the Luenberger-like observer outputs. According to equation (13), the object function of the parameter optimization problem is

$$
\begin{array}{ll}
\min & \left(\sum_{i=1}^{3} \rho_{y_{i} y_{i}}-3\right) \\
\text { s.t. } & {\left[\begin{array}{c}
0 \leq \theta_{1} \leq 1 \\
-1 \leq \theta_{2} \leq 1 \\
0 \leq \theta_{3} \leq 1 \\
0 \leq \theta_{4} \leq 1 \\
0 \leq \theta_{5} \leq 1
\end{array}\right] .}
\end{array}
$$




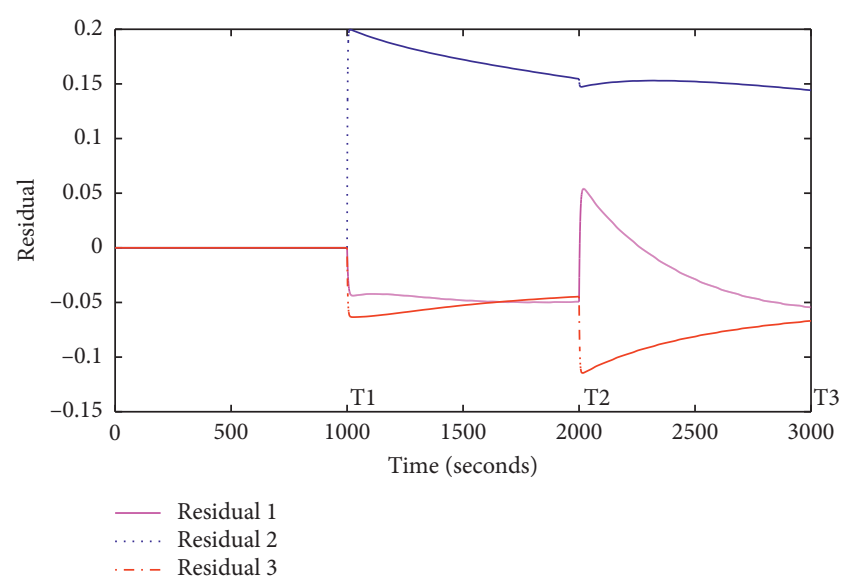

Figure 7: The residual output (time window $=1000, \mathrm{SNR}=50$ ).

\subsection{Experiments and Numerical Results}

5.3.1. The Results in the Absence of Noise. In order to verify the effectiveness of the proposed approach in the paper, the noise-free case is first examined with the aid of the threetank system. In the system, five parameters can constitute 31 fault modes. Considering that the essence of any fault mode is to estimate the coordinates of parameters in the parameter space, only five typical modes are selected for verification of the proposed approach. Five experiments were conducted for five fault modes. The process parameters of the system are given in Table 1 . The diagnosis results with different fault dimension are shown in Table 2.

5.3.2. The Results in the Presence of Noise. Now, the output signal is assumed to be corrupted by noise. If the noise is relatively high compared to the effect of fault on the output, then the correlation coefficient will decrease. Thus, performance of fault identification will degrade. Figure 3 shows the relationship between the correlation coefficient and signal to noise ratio (SNR). The values of the estimated parameters under different output SNR are shown in Table 3, which indicates that the higher the SNR, the better the estimation performance.

From Figure 3, when SNR $>30$, the correlation coefficient is close to 1 and changes slowly. When $\mathrm{SNR}<30$, the more the SNR, the larger the correlation coefficient. All the implementations were made in MATLAB R2016b. Figures 4 and 5 show the parameter identification results with different SNR. Comparing to Figure 5, Figure 4 shows the degradation of parameter estimation performance in the low SNR. Figure 6 shows the real output of the three tanks and the estimated output. From Figure 6, it can be seen that when the correlation coefficient is 3 , the two curves almost overlap. Figure 7 shows the residual changes when the faults occur at 1000 and 2000 seconds.

\section{Conclusion}

In this paper, the diagnosis system follows a general modelbased architecture where the output signal correlation coefficient is used to detect inconsistencies between model predictions and sensor data. The fault detection system and fault identification system are designed. In the fault identification system, a variable parameter model is used to simulate the real system. The output correlation coefficient between the fault detection system and the fault identification system is formulated as an optimization problem. It is solved by evolutionary algorithms to estimate fault parameters. By integrated estimation of all parameters of the system, the coordinates of the fault point in the parameter space can be obtained and the faults severity and mode of the fault (single fault or multiple faults) can be determined. Comparing with common residual-based methods, e.g., filter and adaptive observer methods, the proposed method reduces the number and the complexity of the observer for the estimation of high-dimensional parameter. It overcomes the problem of combinatorial explosion of fault modes and simplifies the fault diagnosis structure. To our knowledge, there are few papers on the adaptive isolation and identification mechanism which combine the observer and intelligent optimization algorithms. Using the powerful global search ability of an evolutionary algorithm, the proposed method is more beneficial to overcome local optimality comparing with the traditional adaptive search mechanism based on parameter gradient change.

\section{Data Availability}

Since the experimental results are obtained by the simulation calculation of the mathematical model, the paper does not use explicit data. Models and parameters are present in the submission.

\section{Conflicts of Interest}

The authors declare that they have no conflicts of interest.

\section{Acknowledgments}

This work was supported by the Collaborative Foundation of Guizhou Province (nos. 7228[2017], 2154[2019], and 7434 [2016]), National Natural Science Foundation of China under grants 61963009, 51867006, and 61861007, Guizhou University introduction of talent research projects [2014] 08, Science and Technology Planning Project of Guizhou Province (nos. 2302[2016] and 5781[2018]), and Platform Talent Project of Guizhou Province (no. 5788[2017]).

\section{References}

[1] C. Ding, M. Zhao, J. Lin, and J. Jiao, "Multi-objective iterative optimization algorithm based optimal wavelet filter selection for multi-fault diagnosis of rolling element bearings," ISA Transactions, vol. 88, pp. 199-215, 2019.

[2] O. Prakash, A. K. Samantaray, and R. Bhattacharyya, "Modelbased multi-component adaptive prognosis for hybrid dynamical systems," Control Engineering Practice, vol. 72, pp. 1-18, 2018.

[3] D. Binu and B. S. Kariyappa, "A survey on fault diagnosis of analog circuits: taxonomy and state of the art," 
AEU-International Journal of Electronics and Communications, vol. 73, pp. 68-83, 2017.

[4] M. Mrugalski, M. Luzar, M. Pazera, M. Witczak, and C. Aubrun, "Neural network-based robust actuator fault diagnosis for a non-linear multi-tank system," ISA Transactions, vol. 61, pp. 318-328, 2016.

[5] Y. Wu, Y. Wang, Y. Jiang, and Q. Sun, "Multiple parametric faults diagnosis for power electronic circuits based on hybrid bond graph and genetic algorithm," Measurement, vol. 92, pp. 365-381, 2016.

[6] C. Huang, F. Naghdy, and H. Du, "Observer-based faultTolerant controller for uncertain steer-by-wire systems using the delta operator," IEEE/ASME Transactions on Mechatronics, vol. 23, no. 6, pp. 2587-2598, 2018.

[7] F. Baghernezhad and K. Khorasani, "Computationally intelligent strategies for robust fault detection, isolation, and identification of mobile robots," Neurocomputing, vol. 171, pp. 335-346, 2016.

[8] M. Krysander and E. Frisk, "Sensor placement for fault diagnosis," IEEE Transactions on Systems, Man, and Cybernetics. Part A, Systems and Humans, vol. 38, no. 6, pp. 1-1410, 2008.

[9] D. Jung and E. Frisk, "Residual selection for fault detection and isolation using convex optimization," Automatica, vol. 97, pp. 143-149, 2018.

[10] D. Jung, K. Y. Ng, E. Frisk, and M. Krysander, "Combining model-based diagnosis and data-driven anomaly classifiers for fault isolation," Control Engineering Practice, vol. 80, pp. 146-156, 2018.

[11] K. Vijayaraghavan and A. Valibeygi, "Adaptive nonlinear observer for state and unknown parameter estimation in noisy systems," International Journal of Control, vol. 89, no. 1, pp. 1-39, 2015.

[12] M. Mohamed, X.-G. Yan, Z. Mao, and B. Jiang, "Adaptive observer design for a class of nonlinear interconnected systems with uncertain time varying parameters," IFAC-PapersOnLine, vol. 50, no. 1, pp. 1421-1426, 2017.

[13] F. Nemati, S. M. Safavi Hamami, and A. Zemouche, "A nonlinear observer-based approach to fault detection, isolation and estimation for satellite formation flight application," Automatica, vol. 107, pp. 474-482, 2019.

[14] A. Slimani, P. Ribot, E. Chanthery, and N. Rachedi, "Fusion of model-based and data-based fault diagnosis approaches," IFAC-PapersOnLine, vol. 51, no. 24, pp. 1205-1211, 2018.

[15] W. S. Sakr, R. A. EL-Sehiemy, and A. M. Azmy, "Adaptive differential evolution algorithm for efficient reactive power management," Applied Soft Computing, vol. 53, pp. 336-351, 2017.

[16] Y. Cai, G. Sun, T. Wang, H. Tian, Y. Chen, and J. Wang, "Neighborhood-adaptive differential evolution for global numerical optimization," Applied Soft Computing, vol. 59, pp. 659-706, 2017.

[17] Y. Zhang, Z. Wang, L. Ma, and F. E. Alsaadi, “Annulus-eventbased fault detection, isolation and estimation for multirate time-varying systems: applications to a three-tank system," Journal of Process Control, vol. 75, pp. 48-58, 2019.

[18] J. Li, K. Pan, and Q. Su, "Sensor fault detection and estimation for switched power electronics systems based on sliding mode observer," Applied Mathematics and Computation, vol. 353, pp. 282-294, 2019.

[19] X. Xu, W. Wang, N. Zou, L. Chen, and X. Cui, "A comparative study of sensor fault diagnosis methods based on observer for ECAS system," Mechanical Systems and Signal Processing, vol. 87, pp. 169-183, 2017.
[20] K. Zhang, B. Jiang, X.-G. Yan, and Z. Mao, "Incipient sensor fault estimation and accommodation for inverter devices in electric railway traction systems," International Journal of Adaptive Control and Signal Processing, vol. 31, no. 5, pp. 785-804, 2017.

[21] H.-J. Ma and G.-H. Yang, "Simultaneous fault diagnosis for robot manipulators with actuator and sensor faults," Information Sciences, vol. 366, pp. 12-30, 2016.

[22] S. X. Ding, Model-based Fault Diagnosis Techniques: Design Schemes, Algorithms and Tools, Springer, Berlin, Germany, 2nd edition, 2013. 